\title{
Optical polarization observations in Hogg 22 and NGC 6204^
}

\author{
R. Martínez, M. M. Vergne ${ }^{\star \star}$, and C. Feinstein ${ }^{\star \star}$
}

\begin{abstract}
Facultad de Ciencias Astronómicas y Geofísicas, Observatorio Astronómico, Paseo del Bosque, 1900 La Plata, Argentina Instituto de Astrofísica de La Plata, CONICET, Argentina
\end{abstract}

Received 5 November 2003 / Accepted 2 December 2003

\begin{abstract}
We present new (UBVRI) multicolor linear polarimetric data for 22 of the brightest stars in the area of the open clusters Hogg 22 and NGC 6204 to study the properties of the ISM (interstellar medium) toward these clusters and between them. The new data were incorporated in our data set of previous observations (Waldhausen et al. 1999), resulting in 28 observed stars in the region. Our data yield for NGC 6204 a mean polarization percentage of $P_{\lambda_{\max }} \sim 1.8 \%$, close to the polarization value produced by the ISM with normal efficiency $\left(P_{\lambda_{\max }} \sim 5 E_{B-V}\right)$ with a color excess of $E_{B-V}=0.51$. Meanwhile for Hogg 22 , located behind NGC 6204, the mean polarization is $P_{\lambda_{\max }} \sim 2.15 \%$, lower than the expected value for the observed color excess of $E_{B-V}=0.68$ (Forbes et al. 1996) and the average efficiency of polarization for the interstellar dust. The mean angle of the polarization vectors of Hogg 22 is $\theta=44^{\circ}$, , which agrees with the expected angle produce by dust particles aligned in the direction of the Galactic Plane $\left(\theta=48^{\circ}\right)$, while for NGC 6204 a lower value, $\theta=33^{\circ}$.7, was found. Therefore, we believe that Hogg 22 is depolarized by the same dust that is polarizing NGC 6204, due to different orientations of the dust particles (and magnetic fields) that polarize the starlight.
\end{abstract}

Key words. ISM: dust, extinction - Galaxy: open clusters and associations: individual: NGC 6204 Galaxy: open clusters and associations: individual: Hogg 22

\section{Introduction}

The polarimetric technique is a very useful tool to get significant information (magnetic field direction, $\lambda_{\max }, P_{\max }$, etc.) from the dust located in front of a luminous object. Young open clusters are very good candidates to carry out polarimetric observations, because previous photometric and spectroscopic studies of these clusters have given detailed information on the color and luminosity of the main sequence stars of the cluster. So, we can compute the physical parameters of the clusters (age, distance, extinction, membership, etc.) and then, with the polarimetric data, we can study the location, size, and efficiency of the dust grains to polarize the starlight and the different directions of the galactic magnetic field in the line of sight to the cluster. As the open clusters are also spread within an area, we can analyze the evolution of the physical parameters of the dust all over the region.

In this framework, we are conducting systematic polarimetric observations in a large number of galactic open clusters. Following our ongoing program, we have performed polarimetry in the open cluster Hogg 22.

Send offprint requests to: $\mathrm{C}$. Feinstein, e-mail: cfeinstein@fcaglp.edu.ar

* Based on observations obtanined at Complejo Astronómico El Leoncito (CASLEO), operated under agreement between the CONICET and the National Universities of La Plata, Córdoba, and San Juan, Argentina.

$\star \star$ Member of Carrera del Investigador Científico, CONICET, Argentina.
Hogg 22 is a compact open cluster in the Ara region ( $l=$ $\left.338^{\circ} 6, b=-1^{\circ} .1\right)$. This object is behind NGC 6204, and both of them (NGC 6204 and Hogg 22) appear as a single group. Hogg (1965) first identified this clump of stars as a distinct object.

The first photometric data were obtained by Whiteoak (1963). Forbes \& Short (1996) performed $U B V$ photoelectric photometry in NGC 6204 and Hogg 22. Most of their data separate into two distinct groups, one comprising stars with colors corresponding to spectral types later than about B5, with a mean reddening $E_{B-V} \simeq 0.51 \pm 0.07$ (NGC 6204) and a second group whose colors imply spectral types as early as about O8, with $E_{B-V} \simeq 0.68 \pm 0.03$ (Hogg 22). In the results obtained by Forbes \& Short (1996) there is a very clear contrast between the two clusters. According to Maeder \& Meynet (1988) evolutionary tracks, Hogg 22 is a much younger group than NGC 6204, their ages being of $5 \pm 2 \mathrm{Myr}$ and $125 \pm 25 \mathrm{Myr}$, respectively. The distances estimated by Forbes \& Short (1996) are $1180 \pm$ 50 pc for NGC 6204 and $2240 \pm 145$ pc for Hogg 22 .

Waldhausen et al. (1999, hereafter WMF) carried out a multicolor polarimetric study in the ARA OB1 region, including the clusters NGC 6167, 6193 and 6204. According to their data, NGC 6204 shows a polarization value of $1.7 \%$ and the distribution in angles of the polarization vectors seems to be Gaussian, with a more likely value of $36^{\circ} .2$.

In the present study, we report the results of the multicolor $(U B V R I)$ measurements of polarization in Hogg 22 and NGC 6204, using also the observations obtained by WMF for the cluster NGC 6204. We studied the characteristics $\left(P_{\max }\right.$, 
Table 1. Polarimetric observations of stars in HOGG 22.

\begin{tabular}{|c|c|c|}
\hline Filter & $\begin{array}{c}P_{\lambda} \pm \epsilon_{\mathrm{P}} \\
\%\end{array}$ & $\theta_{\lambda} \pm \epsilon_{\theta}$ \\
\hline \multicolumn{3}{|c|}{ Star 10} \\
\hline$U$ & $1.66 \pm 0.27$ & $52.8 \pm 4.5$ \\
\hline$B$ & $2.23 \pm 0.25$ & $46.5 \pm 3.2$ \\
\hline$V$ & $1.94 \pm 0.10$ & $48.6 \pm 1.5$ \\
\hline$R$ & $1.90 \pm 0.14$ & $45.5 \pm 2.2$ \\
\hline I & $1.64 \pm 0.19$ & $49.7 \pm 3.3$ \\
\hline \multicolumn{3}{|c|}{ Star 47} \\
\hline$U$ & $1.57 \pm 0.18$ & $41.3 \pm 3.3$ \\
\hline$B$ & $1.89 \pm 0.15$ & $43.0 \pm 2.3$ \\
\hline$V$ & $1.94 \pm 0.09$ & $45.2 \pm 1.3$ \\
\hline$R$ & $2.01 \pm 0.07$ & $45.6 \pm 1.0$ \\
\hline I & $1.64 \pm 0.13$ & $45.6 \pm 2.3$ \\
\hline \multicolumn{3}{|c|}{ Star 49} \\
\hline$U$ & $1.88 \pm 0.16$ & $40.7 \pm 2.4$ \\
\hline$B$ & $2.06 \pm 0.17$ & $41.9 \pm 2.4$ \\
\hline$V$ & $1.90 \pm 0.17$ & $44.4 \pm 2.6$ \\
\hline$R$ & $1.93 \pm 0.14$ & $43.2 \pm 2.1$ \\
\hline$I$ & $1.75 \pm 0.19$ & $43.1 \pm 3.0$ \\
\hline \multicolumn{3}{|c|}{ Star 59} \\
\hline$U$ & $2.34 \pm 0.29$ & $40.5 \pm 3.6$ \\
\hline$B$ & $2.46 \pm 0.22$ & $35.2 \pm 2.6$ \\
\hline$V$ & $2.21 \pm 0.17$ & $40.5 \pm 2.2$ \\
\hline$R$ & $2.37 \pm 0.12$ & $40.4 \pm 1.4$ \\
\hline$I$ & $1.58 \pm 0.28$ & $35.1 \pm 5.1$ \\
\hline \multicolumn{3}{|c|}{ Star 65} \\
\hline$U$ & $0.49 \pm 0.10$ & $34.3 \pm 6.1$ \\
\hline$B$ & $0.65 \pm 0.08$ & $33.4 \pm 3.4$ \\
\hline V & $0.67 \pm 0.05$ & $33.1 \pm 2.3$ \\
\hline$R$ & $0.67 \pm 0.05$ & $33.9 \pm 2.3$ \\
\hline$I$ & $0.73 \pm 0.12$ & $28.9 \pm 4.8$ \\
\hline
\end{tabular}

$\lambda_{\max }$, polarization efficiency, etc.) of the dust located in the line of sight to the region where both clusters are situated. In the next sections, we will discuss the new observations in the region, the data calibrations and the results in terms of each cluster and its extinction.

\section{Observations and data reduction}

Data on linear optical multicolor polarimetry were obtained during two observing runs at the Complejo Astronómico El Leoncito (CASLEO) in San Juan, Argentina, in 2002 (August 6-11) and in 2003 (April 29-May 2). The observations were carried out using the Torino five-channel photopolarimeter attached to the $2.15 \mathrm{~m}$ telescope. All the stars were observed simultaneously through the Johnson-Cousins broad band UBVRI filters $\left(\lambda_{U \mathrm{eff}}=0.360 \mu \mathrm{m}, \lambda_{B \mathrm{eff}}=0.440 \mu \mathrm{m}\right.$, $\left.\lambda_{V \text { eff }}=0.530 \mu \mathrm{m}, \lambda_{\text {Reff }}=0.690 \mu \mathrm{m}, \lambda_{\text {Ieff }}=0.830 \mu \mathrm{m}\right)$. Standard stars for null polarization and for the zero point of the polarization position angle were observed several times each night for calibration purposes.

The polarimetric observations are listed in Table 1 (Hogg 22) and Table 2 (NGC 6204) which show the stellar
Table 1. continued.

\begin{tabular}{|c|c|c|}
\hline Filter & $\begin{array}{c}P_{\lambda} \pm \epsilon_{\mathrm{P}} \\
\%\end{array}$ & $\begin{array}{c}\theta_{\lambda} \pm \epsilon_{\theta} \\
\circ\end{array}$ \\
\hline \multicolumn{3}{|c|}{ Star 67} \\
\hline$U$ & $2.27 \pm 0.19$ & $59.5 \pm 2.4$ \\
\hline$B$ & $2.54 \pm 0.18$ & $53.3 \pm 2.1$ \\
\hline$V$ & $2.46 \pm 0.16$ & $53.4 \pm 1.9$ \\
\hline$R$ & $2.64 \pm 0.11$ & $52.8 \pm 1.2$ \\
\hline$I$ & $2.23 \pm 0.11$ & $50.3 \pm 1.4$ \\
\hline \multicolumn{3}{|c|}{ Star 69} \\
\hline$U$ & $1.24 \pm 0.10$ & $49.2 \pm 2.3$ \\
\hline$B$ & $1.56 \pm 0.13$ & $45.2 \pm 2.4$ \\
\hline$V$ & $1.69 \pm 0.09$ & $46.5 \pm 1.5$ \\
\hline$R$ & $1.74 \pm 0.08$ & $45.6 \pm 1.3$ \\
\hline$I$ & $1.60 \pm 0.16$ & $45.6 \pm 2.8$ \\
\hline \multicolumn{3}{|c|}{ Star 71} \\
\hline$U$ & $1.66 \pm 0.30$ & $36.0 \pm 5.0$ \\
\hline$B$ & $2.04 \pm 0.27$ & $44.8 \pm 3.8$ \\
\hline$V$ & $2.15 \pm 0.23$ & $43.4 \pm 3.0$ \\
\hline$R$ & $2.22 \pm 0.16$ & $44.7 \pm 2.1$ \\
\hline$I$ & $1.81 \pm 0.17$ & $42.2 \pm 2.7$ \\
\hline \multicolumn{3}{|c|}{ Star 72} \\
\hline$B$ & $1.74 \pm 0.46$ & $44.6 \pm 7.3$ \\
\hline$V$ & $1.74 \pm 0.46$ & $47.6 \pm 7.4$ \\
\hline$R$ & $1.60 \pm 0.45$ & $47.5 \pm 7.9$ \\
\hline \multicolumn{3}{|c|}{ Star 74} \\
\hline$U$ & $1.65 \pm 0.15$ & $46.6 \pm 2.7$ \\
\hline$B$ & $2.16 \pm 0.13$ & $47.5 \pm 1.7$ \\
\hline$V$ & $2.34 \pm 0.17$ & $50.8 \pm 2.1$ \\
\hline$R$ & $2.11 \pm 0.16$ & $53.1 \pm 2.1$ \\
\hline$I$ & $2.03 \pm 0.20$ & $48.5 \pm 2.8$ \\
\hline \multicolumn{3}{|c|}{ Star 75} \\
\hline$B$ & $1.40 \pm 0.32$ & $60.3 \pm 6.4$ \\
\hline$V$ & $1.00 \pm 0.30$ & $57.3 \pm 8.3$ \\
\hline$R$ & $1.46 \pm 0.23$ & $46.6 \pm 4.5$ \\
\hline \multicolumn{3}{|c|}{ Star 77} \\
\hline$U$ & $1.53 \pm 0.23$ & $57.2 \pm 4.3$ \\
\hline$B$ & $1.89 \pm 0.17$ & $51.1 \pm 2.5$ \\
\hline$V$ & $2.40 \pm 0.22$ & $56.1 \pm 2.6$ \\
\hline$R$ & $2.26 \pm 0.19$ & $54.4 \pm 2.4$ \\
\hline$I$ & $2.26 \pm 0.26$ & $56.7 \pm 3.3$ \\
\hline \multicolumn{3}{|c|}{ Star 100} \\
\hline$B$ & $2.67 \pm 0.31$ & $40.8 \pm 3.2$ \\
\hline$V$ & $2.52 \pm 0.22$ & $42.8 \pm 2.5$ \\
\hline$R$ & $2.60 \pm 0.19$ & $42.2 \pm 2.1$ \\
\hline$I$ & $2.28 \pm 0.19$ & $44.0 \pm 2.4$ \\
\hline
\end{tabular}

identification as given by Whiteoak (1963), the average polarization percentage $\left(P_{\lambda}\right)$ and the position angle of the electric vector $\left(\theta_{\lambda}\right)$ through each filter, along with their respective mean errors computed as described by Maronna et al. (1992). Since 
Table 2. Polarimetric observations of stars in NGC 6204.

\begin{tabular}{|c|c|c|}
\hline Filter & $\begin{array}{c}P_{\lambda} \pm \epsilon_{\mathrm{P}} \\
\%\end{array}$ & $\begin{array}{c}\theta_{\lambda} \pm \epsilon_{\theta} \\
\circ\end{array}$ \\
\hline \multicolumn{3}{|l|}{ Star 4} \\
\hline$B$ & $1.58 \pm 0.37$ & $34.1 \pm 6.5$ \\
\hline V & $1.62 \pm 0.44$ & $44.3 \pm 7.6$ \\
\hline$R$ & $1.67 \pm 0.59$ & $50.2 \pm 9.7$ \\
\hline$I$ & $1.90 \pm 0.64$ & $42.8 \pm 9.3$ \\
\hline \multicolumn{3}{|c|}{ Star 14} \\
\hline$U$ & $1.46 \pm 0.40$ & $18.6 \pm 7.5$ \\
\hline B & $1.82 \pm 0.27$ & $36.5 \pm 4.2$ \\
\hline V & $1.78 \pm 0.16$ & $35.7 \pm 2.5$ \\
\hline$R$ & $1.92 \pm 0.17$ & $36.3 \pm 2.6$ \\
\hline$I$ & $1.57 \pm 0.22$ & $41.1 \pm 4.1$ \\
\hline \multicolumn{3}{|c|}{ Star 35} \\
\hline$B$ & $1.74 \pm 0.48$ & $35.5 \pm 7.7$ \\
\hline V & $1.71 \pm 0.31$ & $35.7 \pm 5.2$ \\
\hline$R$ & $1.76 \pm 0.40$ & $33.4 \pm 6.4$ \\
\hline \multicolumn{3}{|c|}{ Star 39} \\
\hline$U$ & $1.09 \pm 0.17$ & $36.6 \pm 4.4$ \\
\hline B & $1.09 \pm 0.17$ & $34.9 \pm 4.5$ \\
\hline V & $1.17 \pm 0.13$ & $40.0 \pm 3.2$ \\
\hline$R$ & $1.00 \pm 0.11$ & $38.1 \pm 3.2$ \\
\hline$I$ & $0.87 \pm 0.18$ & $40.4 \pm 5.9$ \\
\hline \multicolumn{3}{|c|}{ Star 45} \\
\hline$U$ & $1.32 \pm 0.28$ & $45.6 \pm 6.1$ \\
\hline B & $1.74 \pm 0.19$ & $31.4 \pm 3.0$ \\
\hline V & $2.02 \pm 0.12$ & $34.7 \pm 1.7$ \\
\hline$R$ & $2.10 \pm 0.16$ & $32.9 \pm 2.1$ \\
\hline$I$ & $1.60 \pm 0.27$ & $40.9 \pm 4.9$ \\
\hline \multicolumn{3}{|c|}{ Star 54} \\
\hline$U$ & $1.39 \pm 0.23$ & $32.6 \pm 4.8$ \\
\hline$B$ & $1.86 \pm 0.19$ & $31.0 \pm 2.9$ \\
\hline V & $1.87 \pm 0.21$ & $34.5 \pm 3.2$ \\
\hline$R$ & $2.17 \pm 0.20$ & $33.3 \pm 2.7$ \\
\hline$I$ & $1.82 \pm 0.27$ & $34.4 \pm 4.3$ \\
\hline \multicolumn{3}{|c|}{ Star 62} \\
\hline$U$ & $2.11 \pm 0.22$ & $32.1 \pm 3.0$ \\
\hline$B$ & $2.30 \pm 0.10$ & $36.0 \pm 1.3$ \\
\hline$V$ & $2.31 \pm 0.06$ & $36.9 \pm 0.7$ \\
\hline$R$ & $2.24 \pm 0.11$ & $35.7 \pm 1.4$ \\
\hline$I$ & $1.94 \pm 0.15$ & $36.1 \pm 2.1$ \\
\hline \multicolumn{3}{|c|}{ Star 78} \\
\hline$U$ & $1.61 \pm 0.44$ & $43.6 \pm 7.6$ \\
\hline$B$ & $2.10 \pm 0.21$ & $41.1 \pm 2.9$ \\
\hline V & $1.91 \pm 0.17$ & $45.8 \pm 2.6$ \\
\hline$R$ & $2.15 \pm 0.14$ & $40.9 \pm 1.9$ \\
\hline$I$ & $1.95 \pm 0.34$ & $36.7 \pm 4.9$ \\
\hline
\end{tabular}

the Torino photopolarimeter collects photons simultaneously in all the filters $(U B V R I)$, the final data from each filter may be of different quality, especially those in the $U$ band. Therefore, observations whose values are not above the $3 \sigma$ error level were excluded and are not included in Tables 1 and 2.

\section{Results}

Because of the historical confusion between the two clusters, WMF also observed stars of Hogg 22 as if they were members of NGC 6204. These stars are \#65, 67, 69, 71, 72, 74, 77 which we re-observed and included these observations in our data set of Hogg 22, assuming its correct membership. Also we included in our analysis of NGC 6204 the observations from WMF of the stars that are known members of this cluster. The new observations in this work and the data of WMF have been taken with the same telescope and polarimeter, so these data sets are homogeneous.

The sky projection of the $V$-band polarization vectors for the observed stars in NGC 6204 and Hogg 22 are shown in Fig. 1. The dot-dashed line superimposed on the figure is the galactic parallel $b=1.75$ denoting the alignment of the polarization vectors with the projection of the Galactic Plane. This indicates that the dust is aligned by a magnetic field close to the direction of the galactic disk and that the uniform distribution of the vectors suggests that the dust layer producing the polarization is located in an undisturbed place in our Galaxy, too.

Figure 2 (upper panel) displays the $P_{V}(\%)$ vs. $\theta_{V}$ for all the set of observations. This plot shows three different groups of stars. One group is Hogg 22's observations located to the right and another group is the data of NGC 6204 located to the left, with $\theta=40^{\circ}$ the border between the two clusters. Therefore, polarimetry appears to be a good technique to find the correct memberships in this case. Figure 2 also shows a group of stars with polarization values below $1 \%$; these stars are suspected nonmembers and probably nearby objects (\#2, 15, 19, 82 of NGC 6204 and \#65, 75 of Hogg 22).

Unfortunately, for stars \#2, 82, in the cluster NGC 6204, we have not enough photometric data to evaluate if they are members or not. Star \#15 has an excess of $E_{B-V}=0.48$ (Forbes $\&$ Short 1996). If we determine the spectral type, according to the $Q$ parameter (Schmidt-Kaler 1982), we obtain as a possible solution a B7V. Therefore, the computed distance is the same as the cluster and thus this star could be a member of NGC 6204. But the low polarization that is present on this star is a consequence of an intrinsic polarization component found already by WMF. In the case of the star \#19, Moffat \& Vogt (1973) suggested that it was a possible evolved cluster member, located near the Cepheid instability strip. However, the polarization value $\left(P_{V}=0.8 \%\right)$ and the angle $\left(\theta=26^{\circ} .4\right)$ are also too low in comparison to the mean values of the cluster, unless there is some severe depolarization by an intrinsic component.

In the case of Hogg 22, star \#65 is one of the field stars of the cluster that has a low polarization $\left(P_{V}=0.69 \%\right)$ compared to the mean value of Hogg $22\left(P_{V} \sim 2.15 \%\right)$ or the mean value of NGC $6204\left(P_{V} \sim 1.7 \%\right)$, and the polarization angle is smaller than the corresponding mean angle of the cluster. Moffat \& Vogt (1973) considered this object as a foreground star. Later, Forbes \& Short (1996) included it in their sample as a member. If we calculate the $Q$ parameter with the color 


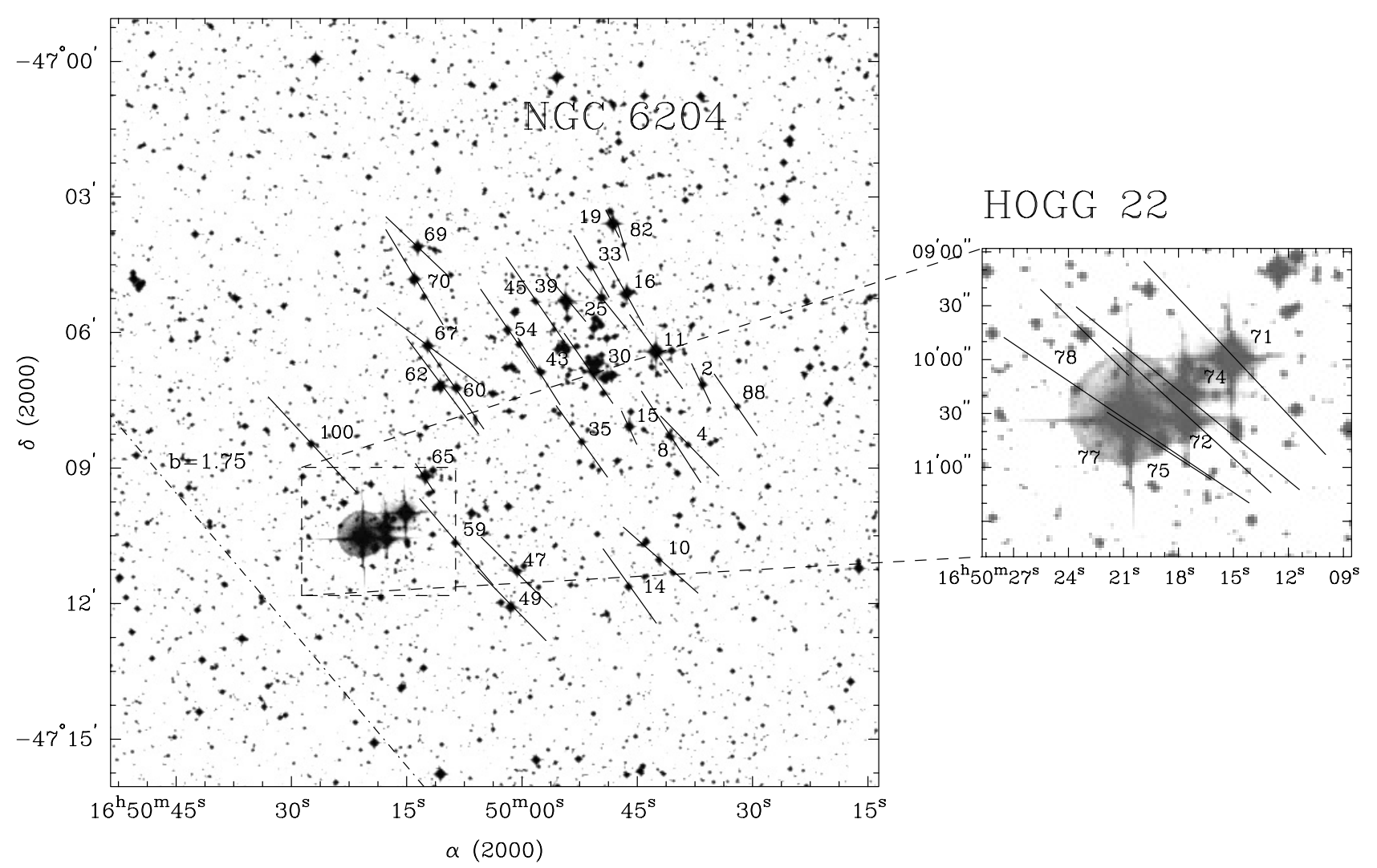

Fig. 1. Projection of the polarization vectors (Johnson $V$ filter) over the sky. The dot-dashed line is the Galactic parallel $b=1.75$.

index $(B-V)$ and $(U-B)$ from the new data of Forbes \& Short (1996), we obtain a spectral type of F5-F8V. So, the star is located close to $190 \mathrm{pc}$, with an $E_{B-V}=0.1$, which explains the low value of the polarimetric vector. Star \#75 shows also a low polarization vector but in this case we think we are dealing with a component of intrinsic polarization. The data for this star is discussed in the next section. We have observed another star of Hogg 22 considered as a nonmember object, star \#72, which was classified as B0.5III by Moffat \& Vogt (1973), therefore, located at $4187.9 \mathrm{pc}$ from the Sun. Therefore, according to the photometric data, this is far more distant than Hogg 22 and of course it is not a member of the cluster, in spite of Moffat \& Vogt (1973) and Forbes \& Short (1996) who included it in their samples. Given the polarization value $\left(P_{V}=1.74 \%\right)$, which is close to the corresponding mean value of Hogg 22, it is possible that there is no interstellar extinction behind Hogg 22, up to $4 \mathrm{kpc}$.

Figure 2 (bottom panel) is the histogram of polarization angles with a Gaussian fit for both clusters. We find that the Gaussian is centered at $44^{\circ} .9$ for Hogg 22 and at $33^{\circ} .7$ for NGC 6204 (the projection of the galactic disk is $48^{\circ}$ ) and has a $F W H M$ of 7.9 and 3.4 , respectively. The center of the Gaussian and the FWHM value of NGC 6204 are different to the values obtained by WMF, because we have established the correct memberships. This result supersedes the one of WMF. The two Gaussian fits re-confirm, with polarization data, that these clusters are two distinct groups. To estimate the quality of the Gaussian fit, a test $\chi^{2}$ was computed for each of these fits (Table 3).
Table 3. Average and $F W H M$ of the main PA for the cluster.

\begin{tabular}{lccc}
\hline \hline Cluster & Average of PA & $F W H M$ of PA & $\chi^{2}$ \\
\hline NGC 6204 & 33.7 & 3.4 & 0.95 \\
Hogg 22 & 44.9 & 8.0 & 0.96 \\
\hline
\end{tabular}

\section{Analysis and discussion}

To analyze the data, the polarimetric observations in the five filters were fitted for each star using Serkowski's law of interstellar polarization (Serkowski 1973). That is:

$P_{\lambda} / P_{\lambda \max }=\mathrm{e}^{-K \ln ^{2}\left(\lambda_{\max } / \lambda\right)}$.

We will assume that, if polarization is produced by aligned interstellar normal dust particles, the observed data (in terms of wavelength, $U B V R I$ ) will then follow (1) and each star will have a $P_{\lambda_{\max }}$ and $\lambda_{\max }$ value.

To perform the fitting we adopted $K=1.66 \lambda_{\max }+0.01$ (Whittet et al. 1992). For each star we also computed the $\sigma_{1}$ parameter (the unit weight error of the fit) to quantify the departure of our data from the "theoretical curve" of Serkowski's law. In our scheme, when a star shows $\sigma_{1}>1.5$, it is indicating the presence of intrinsic stellar polarization. The $\lambda_{\max }$ values can be used to test the origin of the polarization. Those objects which have $\lambda_{\max }$ lower than the average value of the interstellar medium $(0.545 \mu \mathrm{m}$, Serkowski et al. 1975) are candidates to have an intrinsic component of polarization as well (Orsatti et al. 1998). Another criterion to detect intrinsic stellar polarization comes from computing the dispersion of the position 


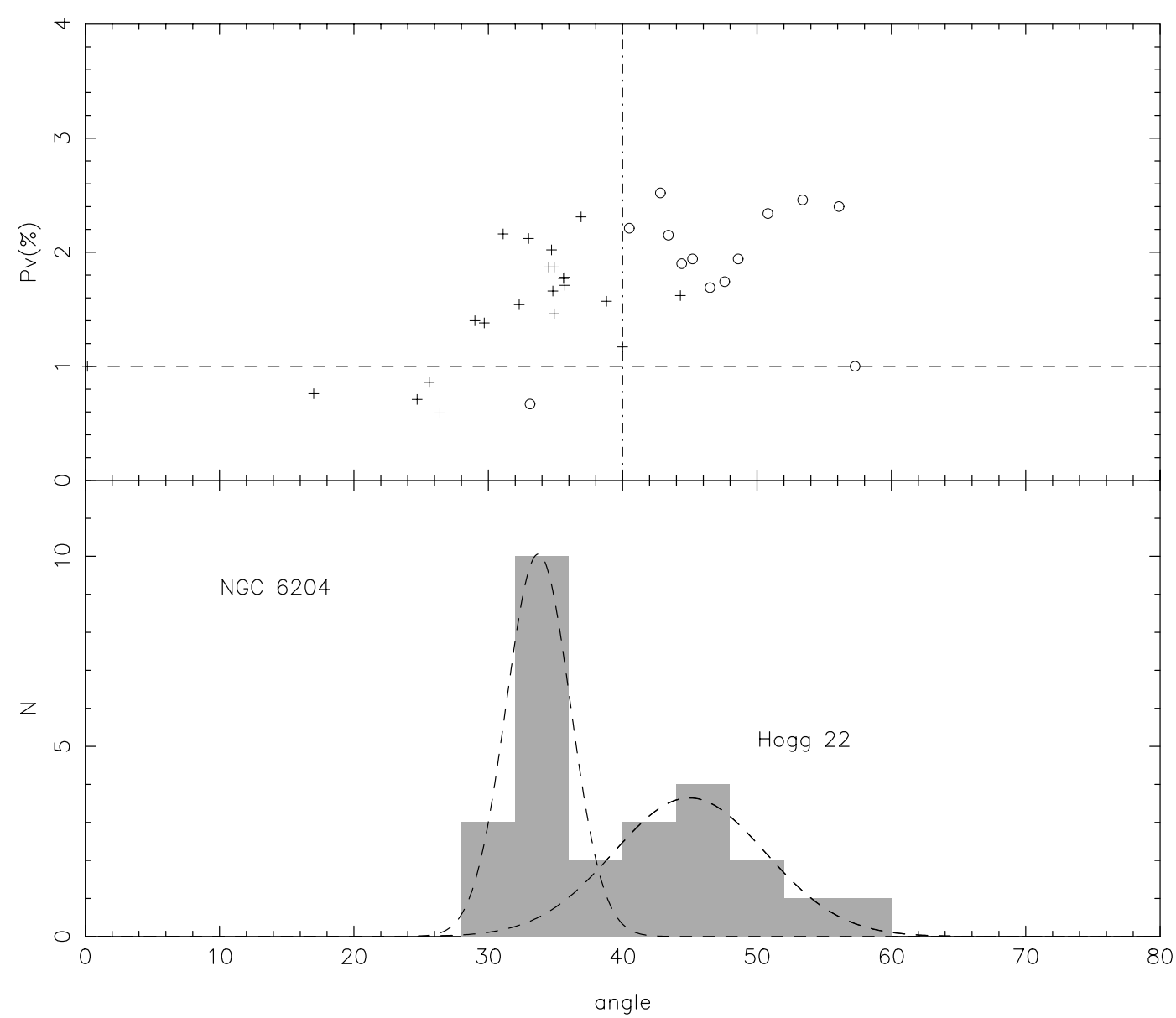

Fig. 2. Upper panel: $P_{V}(\%)$ vs. $\theta_{V}$, below the $1 \%$ line have located most of the non member stars. Crossed are stars belonging to NGC 6204 and open circles are for Hogg 22 stars. Bottom panel: histogram of the polarization angle $\theta_{V}$ for all the observed stars. The continous line is the Gaussian fit to the data of each cluster. This plot shows that the two clusters Hogg 22 and NGC 6204 have on average different polarization angles.

angle for each star normalized by the average of the position angle errors $(\bar{\epsilon})$. The values obtained for $P_{\lambda_{\max }}$, the $\sigma_{1}$ parameter, $\lambda_{\max }$, and $\bar{\epsilon}$ together with the identification of stars are listed in Tables 4 and 5. From the histograms of $P_{\lambda_{\max }}$ for both clusters shown in Fig. 3 (excluding the data of probable nonmembers) we can estimate for Hogg 22 a more likely polarization value of $P_{\lambda_{\max }} \sim 2.2 \%$ and for NGC 6204 of $P_{\lambda_{\max }} \sim 1.8 \%$.

According to the values of the $\sigma_{1}$ parameter, which estimates how suitable the Serkowski law is when is fitted to the observations, we can see that Hogg 22 is well fitted. In all cases the value of $\sigma_{1}$ is smaller than 1.5. Only star \#75 is a candidate for having an intrinsic component of polarization. Unfortunately we only have data for this star in three filters $(B, V, R)$, but these observations show a rotation in the angle of the polarization vectors and a low polarization vector in the $V$ filter. These are indications that the polarization results from a combination of different components with dissimilar polarization angles. Probably there is an initial intrinsic component that is being depolarized by the dust component.

The histograms of all the observed $\lambda_{\max }$ in Fig. 4 confirm that the most probable value of $\lambda_{\max }$ for stars in Hogg 22 and NGC 6204 is similar to the average value for the ISM $(0.545 \mu)$ found by Serkowski et al. (1975).
Table 4. Parameters of the Serkowski fit to the linear polarization data for stars in Hogg 22.

\begin{tabular}{lcccc}
\hline \hline $\begin{array}{l}\text { Stellar } \\
\text { identification }\end{array}$ & $\begin{array}{c}P_{\max } \pm \epsilon_{\mathrm{P}} \\
\%\end{array}$ & $\sigma_{1}$ & $\begin{array}{c}\lambda_{\max } \pm \epsilon_{\lambda_{\max }} \\
\mu \mathrm{m}\end{array}$ \\
\hline 10 & $1.989 \pm 0.059$ & 0.790 & $0.527 \pm 0.036$ & 1.19 \\
47 & $1.991 \pm 0.044$ & 0.856 & $0.583 \pm 0.031$ & 0.60 \\
49 & $2.067 \pm 0.049$ & 0.628 & $0.518 \pm 0.023$ & 0.62 \\
59 & $2.446 \pm 0.158$ & 1.305 & $0.491 \pm 0.060$ & 1.46 \\
65 & $0.693 \pm 0.022$ & 0.565 & $0.638 \pm 0.042$ & 0.46 \\
67 & $2.637 \pm 0.063$ & 0.895 & $0.553 \pm 0.024$ & 3.30 \\
69 & $1.738 \pm 0.021$ & 0.331 & $0.651 \pm 0.013$ & 0.68 \\
71 & $2.153 \pm 0.062$ & 0.593 & $0.576 \pm 0.032$ & 1.48 \\
72 & $1.752 \pm 0.009$ & 0.030 & $0.498 \pm 0.007$ & 0.26 \\
74 & $2.242 \pm 0.077$ & 0.855 & $0.604 \pm 0.036$ & 2.44 \\
75 & $1.362 \pm 0.306$ & 1.327 & $0.665 \pm 0.346$ & 6.25 \\
77 & $2.336 \pm 0.087$ & 0.640 & $0.701 \pm 0.037$ & 1.60 \\
100 & $2.658 \pm 0.079$ & 0.572 & $0.553 \pm 0.033$ & 0.41 \\
\hline
\end{tabular}

The polarization efficiency of the dust towards these clusters is estimated from the $P_{\lambda_{\max }}$ vs. $E_{B-V}$ individual plots. The color excesses were estimated by Forbes \& Short (1996) and 


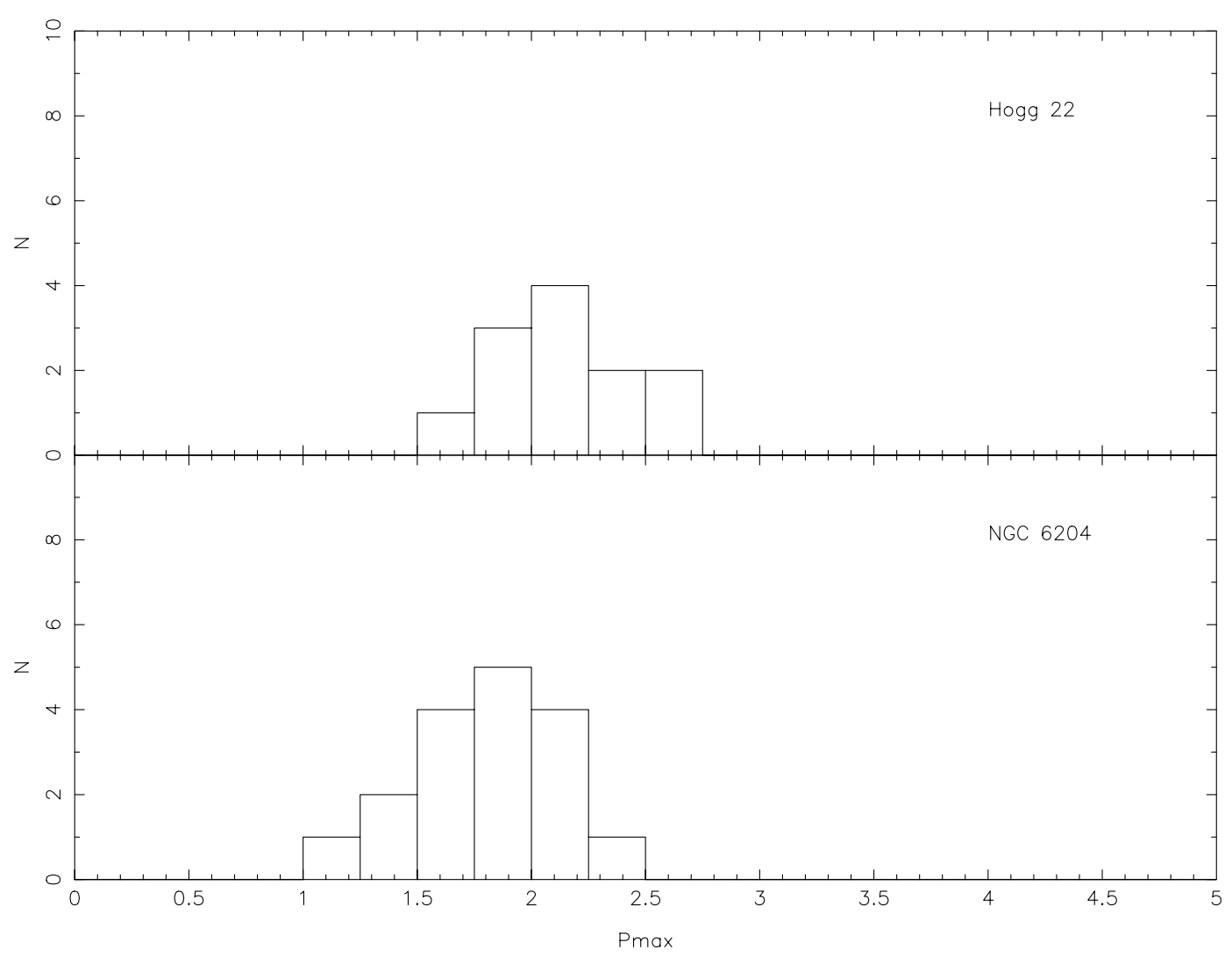

Fig. 3. Histograms of $P_{\lambda \max }$ for each cluster.

Table 5. Parameters of the Serkowski fit to the linear polarization data for stars in NGC 6204.

\begin{tabular}{lcccc}
\hline \hline $\begin{array}{l}\text { Stellar } \\
\text { identification }\end{array}$ & $\begin{array}{c}P_{\max } \pm \epsilon_{\mathrm{P}} \\
\%\end{array}$ & $\sigma_{1}$ & $\begin{array}{c}\lambda_{\max } \pm \epsilon_{\lambda_{\max }} \\
\mu \mathrm{\epsilon}\end{array}$ \\
\hline 4 & $1.797 \pm 0.104$ & 0.308 & $0.674 \pm 0.069$ & 4.31 \\
14 & $1.858 \pm 0.051$ & 0.524 & $0.581 \pm 0.038$ & 3.75 \\
35 & $1.775 \pm 0.059$ & 0.253 & $0.576 \pm 0.064$ & 0.17 \\
39 & $1.148 \pm 0.020$ & 0.248 & $0.473 \pm 0.014$ & 0.82 \\
45 & $2.021 \pm 0.098$ & 0.880 & $0.648 \pm 0.064$ & 2.46 \\
54 & $2.051 \pm 0.087$ & 0.641 & $0.663 \pm 0.044$ & 0.51 \\
62 & $2.336 \pm 0.020$ & 0.447 & $0.529 \pm 0.013$ & 0.50 \\
78 & $2.127 \pm 0.077$ & 0.768 & $0.612 \pm 0.054$ & 1.58 \\
\hline
\end{tabular}

the result is plotted in Fig. 5. Assuming normal interstellar material characterized by $R=3.2$, the empirical upper limit relation for the polarization efficiency given by $P_{\lambda \max }=R A_{\mathrm{v}} \sim$ $9 E_{B-V}$ (Serkowski et al. 1975) is depicted by the continuous line in this figure. Indeed, this line represents the maximum efficiency of polarization produced by the interstellar dust. Likewise, the dashed line $P(\%)=3.5 E(B-V)^{0.8}$, also shown in Fig. 5, represents the new estimate of the average efficiency made by Fosalba et al. (2002). For comparison, the dot-dashed line $P_{\lambda_{\max }} / E_{B-V} \sim 5$ is drawn as a reference of the old accepted determination of the average efficiency.
Figure 5 indicates that the polarization efficiency of NGC 6204 appears to be in good agreement with the Fosalba et al. (2002) relation. In the case of Hogg 22, the efficiency is lower than the value predicted by this relation. In both cases the polarization is low in comparison to the $P_{\lambda_{\max }} / E_{B-V} \sim 5$ line. The diminished polarization, as shown by Hogg 22, could be the result of polarization vectors produced by dust particles oriented by magnetic fields with different angles. While the polarization angle of NGC 6204 displays the mean direction of magnetic field between the Sun and this cluster, the polarization of Hogg 22, which is located far away, shows that behind NGC 6204 there is a dust layer producing polarization with a different angle in comparison to the one produced by dust in front of NGC 6204. Due to this difference in the angle of the polarization vector, the resulting composition appears less polarized. Also Table 4 shows that most of the stars in Hogg 22 have an $\bar{\epsilon}$ parameter higher than the one observed in NGC 6204, and this can be interpreted also as a vector composition, because it is an indication that there is a rotation of the angles between the observations in the different wavelengths.

We suggest that there are two layers of dust clouds, one is lying in front of NGC 6204 and the other component is located behind this cluster and in front of Hogg 22. The first dust cloud is not associated with the cluster NGC 6204, because the nonmember star \#65 seems to be located at $190 \mathrm{pc}$ (while NGC 6204 is placed at $\sim 1200 \mathrm{pc}$ ) and both clusters and star \#65 have the same polarization angle $\left(\theta=33^{\circ}\right)$. But, on the other hand, star \#65 has $P_{V}=0.67 \%$ while NGC 6204 has a mean $P_{V}=1.8 \%$, therefore we think that our first component 


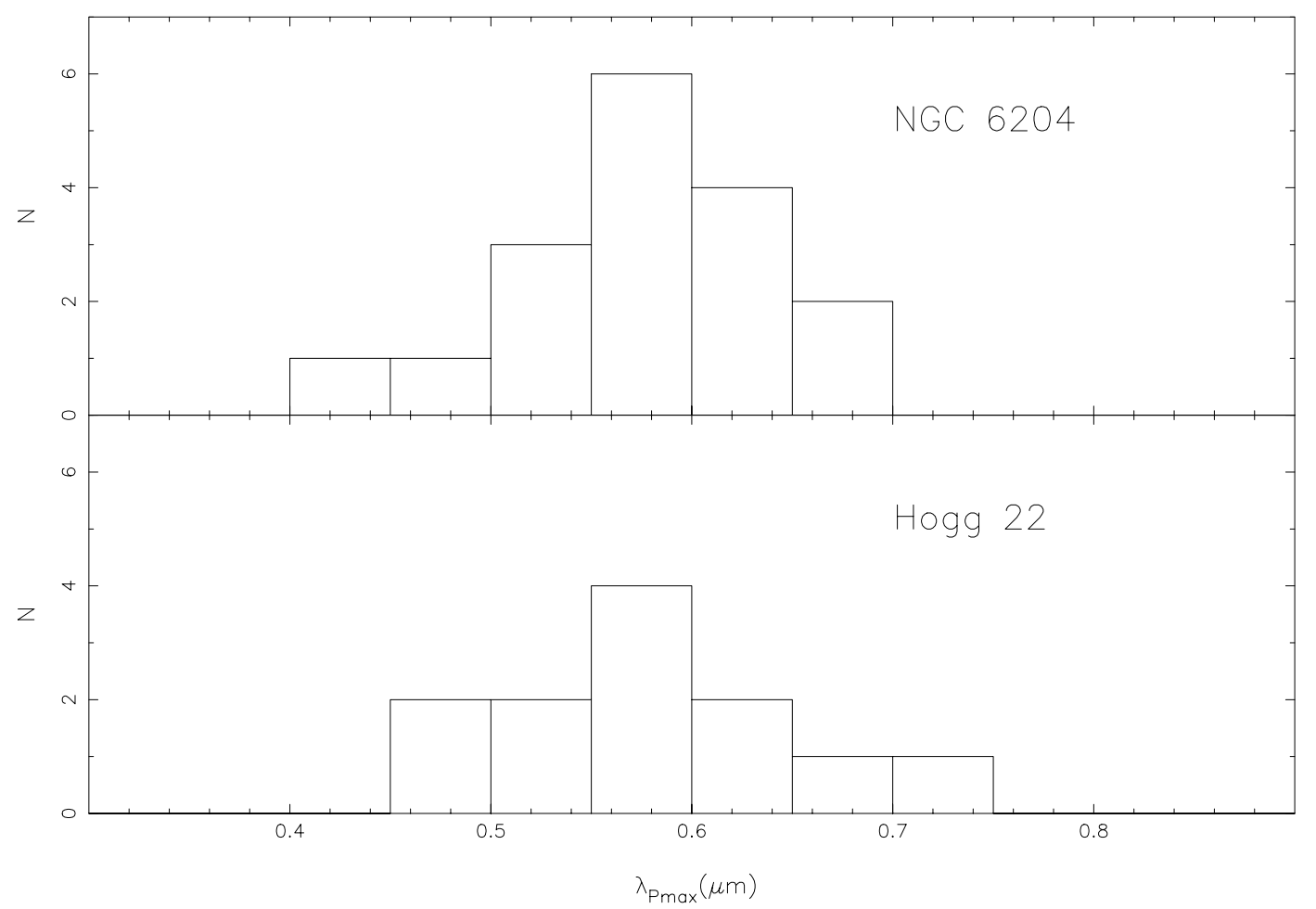

Fig. 4. Histograms of $\lambda_{\max }$ for both clusters. Both plots show a maximum near $0.55 \mu \mathrm{m}$, the canonical value for the interstellar medium.

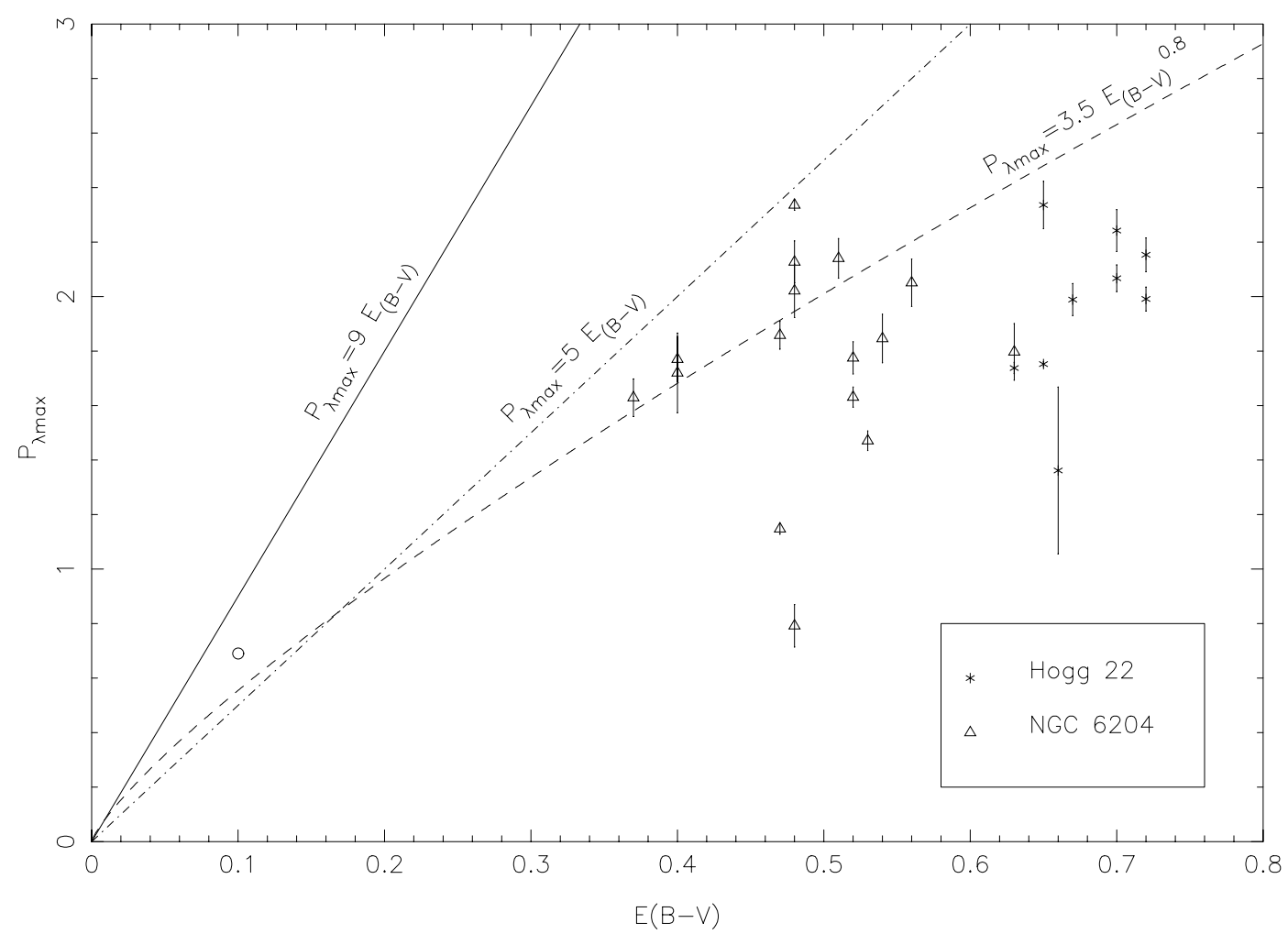

Fig. 5. $P_{\lambda \max }$ vs. $E_{B-V}$. The solid line is $P_{\lambda \max }=9 E_{B-V}$, the dotted line is $P_{\lambda \max }=3.5 E(B-V)^{0.8}$, the dot-dashed line is $P_{\lambda \max }=5 E_{B-V}$. The unfilled asterisks are stars from Hogg 22, the open triangles are data from NGC 6204. The open circle is the nonmember star \#65.

is contributed by clouds in the line of sight, not associated with NGC 6204. So, these clouds are lying in undisturbed zones with the local magnetic field in the direction of the Galactic Plane.
Another possibility is that some of the dust of the second component is within NGC 6204 and does not affect the stars of the cluster located in front. This may explain also the large spread in the $E_{B-V}$ of the individual stars observed in the 


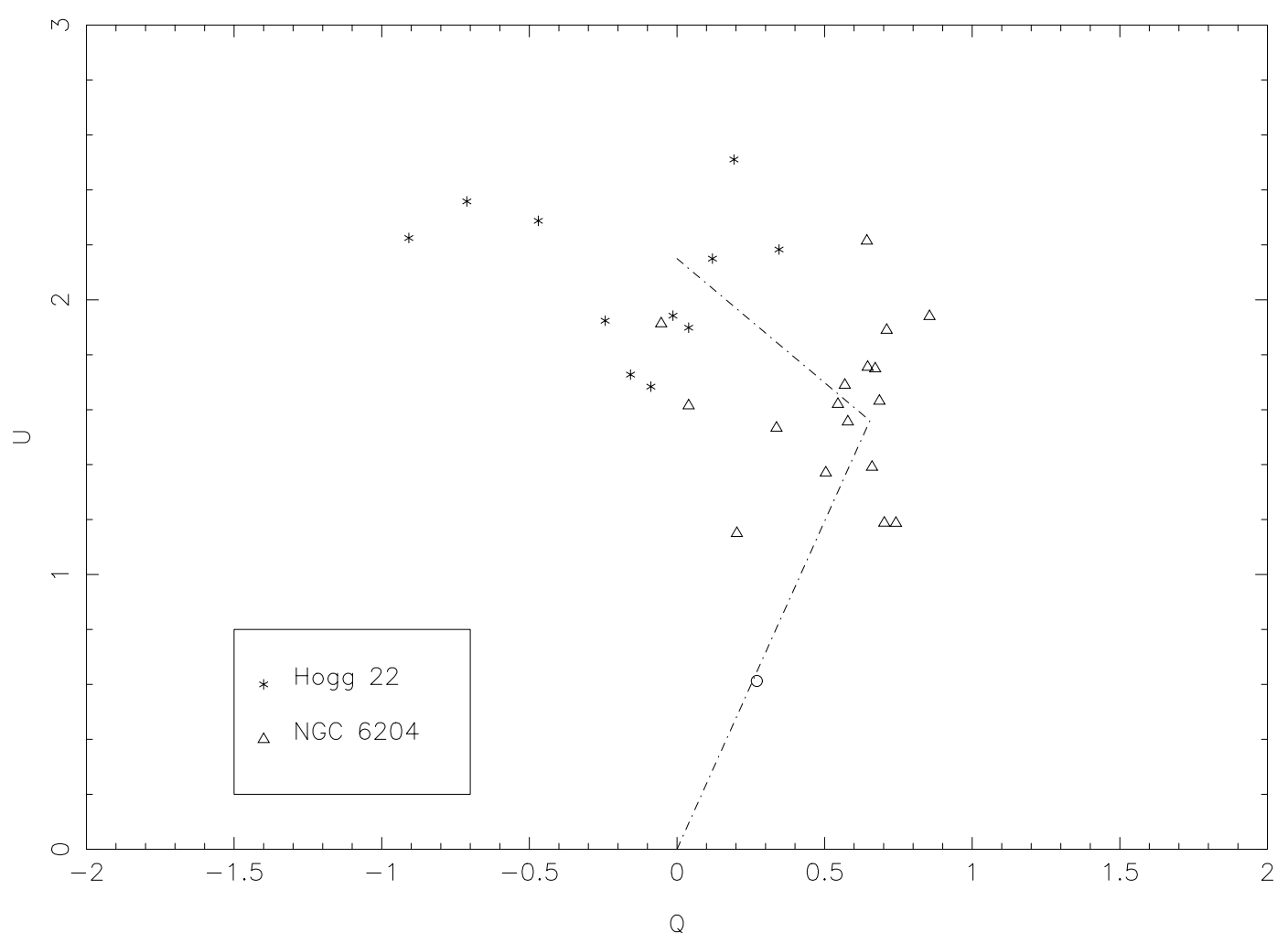

Fig. 6. $U$ vs. $Q$ plot for the stars of NGC 6204 and Hogg 22. Symbols are the same as Fig. 5. The dot-dashed lines show the proposed components of the polarization produced by the dust layer on the line of sight to the clusters.

cluster NGC 6204. Figure 6 shows the plot of the $Q-U$ plane for the observed stars to visualize the decomposition of the polarization of Hogg 22. We have not included any of the low polarization stars that are suspected of being nonmembers. We have drawn in Fig. 6 the main components of polarization for each cluster to show how we interpreted the observations. The first component is from dust that is in front of NGC 6204, but then the observations of Hogg 22 show that there is other component of polarization that makes the polarization angle turns to the right in the $Q-U$ plane (See Fig. 6).

Neckel \& Klare (1980) have computed the interstellar extinction values and distances for more than $11000 \mathrm{O}$ to $\mathrm{F}$ stars in the Milky Way. Their figure 6j(200) shows the variation of $A_{\mathrm{v}}$ with increasing distance in the area of Hogg 22 and NGC 6204. This plot is in good agreement with the results of this work. The plot displays that the absorption increases gradually from the Sun to $1.3 \mathrm{kpc}$, and then remains constant up to $5 \mathrm{Kpc}$. This is compatible with the results of our polarimetry. For example, the low polarization of the foreground star \#65, located at $190 \mathrm{pc}$, has an $E_{B-V}=0.1$ while NGC 6204 is located close to $1.2 \mathrm{kpc}$ and its extinction is $E_{B-V}=0.51$, both of them in very good agreement with Neckel \& Klare's (1980) plot. In the case of Hogg 22 located at $2.2 \mathrm{kpc}$ the plot shows an increase of the extinction up to $A_{\mathrm{V}} \sim 1.8$, also in good agreement with the extinction of the cluster. For the nonmember star \#72, locate behind Hogg 22 ( $d \sim 4 \mathrm{kpc}$ ), that shows a polarimetric vector similar to this cluster (both the polarimectric vector and its angle) the Neckel \& Klare plot confirms that there is a large region of transparency as shown by the polarimetric result.

\section{Summary}

We have observed linear multicolor polarization for a sample of 22 stars for Hogg 22 and NGC 6204. With this new data we increased our set of observations reported previously (WMF). The analysis of all these data shows the following properties:

- By analyzing nonmember stars we find at least two dust layers in the line of sight to NGC 6204. The observations show that both dust layers have their local magnetic field with the same orientation.

- NGC 6204 and Hogg 22 have a different mean angle of polarization and the membership of each star is easily identified by polarimetry.

- The polarimetric vectors in NGC 6204 are not completely orientated with the Galactic Plane, while the vectors in Hogg 22 are.

- The polarimetric efficiency of the dust in front of NGC 6204 is close to the mean value for the Galaxy, while the result obtained for the stars of Hogg 22 is below the expected value.

- The observations of most of the stars in Hogg 22 show characteristics of being depolarized by the composition of the polarimetric vectors produced in front of and behind NGC 6204. This interpretation can explain the low polarization efficiency for Hogg 22.

- A simple model of two different dust components with different orientations of the magnetic field can explain the observations of Hogg 22. 
- Behind Hogg 22 and until star \#72 which is located close to $4 \mathrm{kpc}$ from the Sun, the extinction seems not to increase and remains constant, indicating a large region of transparency.

Acknowledgements. We wish to acknowledge the technical support of CASLEO during our observing runs. Also we want to acknowledge useful discussions with Ana M. Orsatti which is greatly appreciated. We also acknowledge the use of the Torino Photopolarimeter built at Observatorio Astronomico di Torino (Italy) and operated under agreement between Complejo Astronómico El Leoncito and Observatorio Astronomico di Torino.

\section{References}

Forbes, D., \& Short, S. 1996, AJ, 111, 1609

Fosalba, P., Lazarian, A., Prunet, S., \& Tauber, J. A. 2002, ApJ, 564, 762
Johnson, H. L., \& Iriarte, B. 1958, Lowell Obs. Bull. No. 91 Maeder, A., \& Meynet, G. 1988, A\&AS, 76, 411

Moffat, A. F. J., \& Vogt, N. 1973, A\&AS, 10, 135

Maronna, R., Feinstein, C., \& Clocchiatti, A. 1992, A\&A, 260, 525

Neckel, Th., \& Klare, G. 1980, A\&A, 42, 251

Orsatti, A. M., Vega, E., \& Marraco, H. G. 1998, AJ, 116, 226

Serkoswki, S. 1973, in Interstellar Dust and Related topics, ed. J. M. Greenberg, \& H. C. van den Hulst (Dortrecht-Holland: Reidel), Proc. IAU Symp., 52, 145

Serkowski, K., Mathewson, D. L., \& Ford, V. L. 1975, ApJ, 196, 261

Schmidt-Kaler, Th. 1982, Landolt-Bornstein, Numerical Data and Funtional Relationships in Science and Technology, New Series, Group IV, Vol. 9, ed. K. Schaifers, \& H. H. Voigt (Berlin: Springer-Verlag)

Waldhausen, S., Martínez, R., \& Feinstein, C. 1999, AJ, 117, 2882

Whiteoak, J. B. 1963, MNRAS, 125, 91

Whittet, D. C. B., Martin, P. G., Hough, J. H., et al. 1992, ApJ, 386, 562 